\title{
Multicarrier Parameter Optimization in Doubly Selective Fading Channels with LOS Components
}

\author{
Heidi Steendam \\ DIGCOM research group, TELIN Dept., Ghent University \\ Sint-Pietersnieuwstraat 41, 9000 GENT, BELGIUM \\ E-mail: Heidi.Steendam@telin.ugent.be
}

\begin{abstract}
In wireless communications, the channel can often be modelled as a time-selective frequency-selective fading channel with line-of-sight (LOS) component. Although multicarrier systems are developed to cope with the frequency selectivity of the channel, they will suffer from interference caused by the time-varying character of the channel, present in particular in the LOS component, when the number of carriers increases. A proper selection of the system parameters, such as the number of carriers and the length of the cyclic prefix, can alleviate the effect of the doubly-selective channel on the system performance. In this paper, we investigate analytically the effect of the aforementioned system parameters on the performance, and determine the optimum values of the system parameters. Further, we study the effect of deviations from the optimum parameters on the system performance.
\end{abstract}

\section{INTRODUCTION}

In multicarrier systems, the data stream to be transmitted is split into a large number of parallel data streams at a lower rate [1]-[2]. Each of the parallel data streams is transmitted on a different carrier of the multicarrier system. With this operation, the effective symbol duration will be increased with a factor equal to the number of carriers, reducing the effect of the frequency selectiveness of the channel. By inserting a cyclic prefix [3]-[4], a further reduction of the interference caused by frequency selectivity is obtained; moreover, all interference is counteracted when the length of the cyclic prefix is larger than the maximum delay spread of the channel. However, the use of a cyclic prefix reduces the power efficiency and the effective throughput, such that the prefix must be kept small as compared to the duration of the multicarrier symbol, which is proportional to the number of carriers. Hence, from the frequency-selectivity point of view, the cyclic prefix must be chosen larger than the maximum delay spread, and the number of carriers must be selected as large as possible to keep the loss in power efficiency and effective throughput within reasonable margins.

However, the use of a large number of carriers results in very long multicarrier symbols [5]. This makes the multicarrier system more sensitive to the time-selectivity of the channel. Therefore, from the time-selectivity point of view, the number of carriers must be chosen sufficiently small. In a doublyselective channel therefore a compromise must be made between the alleviation of the effects of the time-selectivity and the frequency-selectivity of the channel.

In the literature, the optimization of the system parameters is almost never done jointly: in [3]-[4], the effect of the cyclic prefix length on the performance is investigated in for a given number of carriers, whereas in [5], the effect of the number of carriers is studied for a sufficient cyclic prefix length. Only in [6], the system parameters are jointly optimized for fading channels without LOS component. This paper extends the results obtained in [6] to channels with LOS component. Further, in this paper, we investigate the effect of deviations

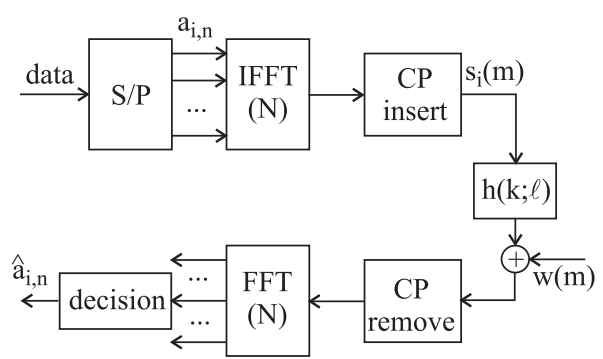

Fig. 1. The multicarrier transceiver.

from the optimum parameters on the system performance, which has not been considered in [6].

\section{SYSTEM DESCRIPTION}

In figure 1, the conceptual block diagram of a multicarrier system is shown. For the sake of notational simplicity, an OFDM system is considered. However, the analysis can easily be extended to other multicarrier systems, e.g. MC-CDMA, MC-DS-CDMA... The data stream to be transmitted is serialto-parallel converted into $N$ lower rate data streams $\left\{a_{i, n}\right\}$, where $a_{i, n}$ is the $i$ th symbol of the $n$th data stream, $n=$ $0, \ldots, N-1$. The data symbols $a_{i, n}$ are transmitted on the different carriers of the multicarrier system using an inverse fast Fourier transform (IFFT). To cope with the presence of a dispersive channel, a cyclic prefix of length $\nu$ samples is inserted, resulting in the time-domain samples $s_{i}(m)$ :

$s_{i}(m)=\sqrt{\frac{E_{s}}{N+\nu}} \sum_{n=0}^{N-1} a_{i, n} e^{j 2 \pi \frac{n m}{N}} \quad m=-\nu, \ldots, N-1$

where the data symbols are assumed to be statistically independent and have unit average energy, i.e. $E\left[a_{i, n} a_{i^{\prime}, n^{\prime}}^{*}\right]=$ $\delta_{i, i^{\prime}} \delta_{n, n^{\prime}}$, and the transmitted energy per symbol is equal to $E_{s}$.

The time-domain samples (1) are transmitted over a doublyselective fading channel. The channel is modelled as a tapped delay line with channel coefficients $h(k ; \ell)$. We assume that the channel consists of a line-of-sight (LOS) component $h_{L O S}(k ; \ell)$ and a zero-mean multipath fading contribution $h_{M P}(k ; \ell)$, i.e. $h(k ; \ell)=h_{L O S}(k ; \ell)+h_{M P}(k ; \ell)$. The coefficients of the LOS component are given by $h_{L O S}(k ; \ell)=$ $\alpha e^{-j \phi(\ell)} \delta(k)$, where the quasi-static amplitude $\alpha$ is assumed to be constant over a number of multicarrier symbols, and $\phi(\ell)$ is a time-varying phase that depends on the time-selectivity of the channel. The channel coefficients $h_{M P}(k ; \ell)$ of the multipath component are assumed to be zero-mean Gaussian distributed according to the wide-sense stationary uncorrelated scattering (WSSUS) model of Bello [7] with autocorrelation 
function $R_{M P}(k ; \ell)$

$$
E\left[h_{M P}\left(k_{1} ; \ell_{1}\right) h_{M P}^{*}\left(k_{2} ; \ell_{2}\right)\right]=\delta\left(\ell_{1}-\ell_{2}\right) R_{M P}\left(k_{1}-k_{2} ; \ell_{1}\right) .
$$

Let us define $P_{L O S}$ and $P_{M P}$ as the energy contained in the impulse response corresponding to the LOS and multipath component, respectively:

$$
\begin{aligned}
& P_{L O S}=|\alpha|^{2} \\
& P_{M P}=\sum_{k=-\infty}^{+\infty} R_{M P}(k ; 0) .
\end{aligned}
$$

We define the ratio $\kappa=P_{L O S} / P_{M P}$ as the ratio of the energy contained in the impulse response of the LOS component to the one of the multipath component. Without loss of generality, we can assume that $P_{L O S}+P_{M P}=1 .^{1}$ The output of the channel is disturbed by complex-valued additive white Gaussian noise $w(m)$, with zero mean and power spectral density $N_{0}$

Without loss of generality, we concentrate on the detection of the data symbols transmitted during the multicarrier block with time index $i=0$. From the $N+\nu$ samples belonging to the considered multicarrier block, the receiver removes the $\nu$ samples corresponding to the cyclic prefix. The remaining $N$ samples are then applied to a fast Fourier transform (FFT). The $n$th output of the FFT can be written as, assuming that all carriers are modulated,

$$
z_{n}=\sqrt{E_{s}} \sqrt{\frac{N}{N+\nu}} \sum_{i=-\infty}^{+\infty} \sum_{n^{\prime}=0}^{N-1} a_{i, n^{\prime}} \gamma_{i}\left(n, n^{\prime}\right)+W_{n}
$$

where

$$
\gamma_{i}\left(n, n^{\prime}\right)=\frac{1}{N} \sum_{m=-\nu}^{N-1} \sum_{k=0}^{N-1} h(k-m-i(N+\nu) ; k) e^{-j 2 \pi \frac{k n-m n^{\prime}}{N}}
$$

and the noise contribution is given by

$$
W_{n}=\frac{1}{\sqrt{N}} \sum_{k=0}^{N-1} w(k) e^{-j 2 \pi \frac{k n}{N}} .
$$

In the presence of a fading channel, in general $\gamma_{i}\left(n, n^{\prime}\right) \neq 0$ for $n^{\prime} \neq n$ and/or $i \neq 0$. Hence, the fading channel will cause interference. The power at the $n$th FFT output can be decomposed in $P(n)=\frac{N}{N+\nu} E_{s}\left(P_{U}(n)+P_{I C I}(n)+\right.$ $\left.P_{I S I}(n)\right)+N_{0}$. The useful power $P_{U}(n)$ corresponds to the contribution of the useful symbol $a_{0, n}$. The intercarrier interference (ICI) power $P_{I C I}(n)$ consists of the contributions of the data symbols transmitted on the other carriers during the considered multicarrier symbol $\left(i=0, n^{\prime} \neq n\right)$. The intersymbol interference (ISI) power $P_{I S I}$ is the contribution of the data symbols transmitted during other multicarrier blocks $(i \neq 0)$. The last contribution is the additive noise power $N_{0}$. The powers of the useful component, the ICI and ISI are given by

$$
\begin{gathered}
P_{U}(n)=E\left[\left|\gamma_{0}(n, n)\right|^{2}\right] \\
P_{I C I}(n)=\sum_{n^{\prime}=0, n^{\prime} \neq n}^{N-1} E\left[\left|\gamma_{0}\left(n, n^{\prime}\right)\right|^{2}\right]
\end{gathered}
$$

${ }^{1}$ Under this assumption, it follows that $P_{L O S}=\frac{\kappa}{1+\kappa}$ and $P_{M P}=\frac{1}{1+\kappa}$.

$$
P_{I S I}(n)=\sum_{i=-\infty, i \neq 0}^{+\infty} \sum_{n^{\prime}=0}^{N-1} E\left[\left|\gamma_{i}\left(n, n^{\prime}\right)\right|^{2}\right] .
$$

It can easily be verified that the useful power (7) and interference powers (8) and (9) are independent of the carrier index $n$. Hence, in the following, we drop the carrier index.

As a performance measure, we use the signal to interference and noise ratio (SINR) at the output of the FFT, defined as the ratio of the useful power to the sum of the powers of the interference and noise.

$$
S I N R=\frac{\frac{N}{N+\nu} E_{s} P_{U}}{\frac{N}{N+\nu} E_{s}\left(P_{I C I}+P_{I S I}\right)+N_{0}}
$$

In the presence of a fading channel, the SINR is degraded as compared to the SINR in the case of an AWGN channel and in the absence of a cyclic prefix, which equals $S I N R_{A W G N}=$ $E_{s} / N_{0}$. The degradation (in $\mathrm{dB}$ ) of the SINR, caused by the fading channel and the presence of a cyclic prefix, is given by

$$
\begin{aligned}
D e g & =-10 \log \left(\frac{N}{N+\nu} P_{U}\right) \\
& +10 \log \left(1+\frac{E_{s}}{N_{0}} \frac{N}{N+\nu}\left(P_{I C I}+P_{I S I}\right)\right)
\end{aligned}
$$

To simplify the expressions (7)-(9), we define similarly as in [6] the following two-dimensional weight function

$$
w(q ; r)=\frac{1}{N} \begin{cases}N-|r| & 0 \leq q \leq \nu \\ N-q+\nu-|r| & 0 \leq|r| \leq N \\ & 0 \leq q \leq N+\nu \\ N+q-|r| & 0 \leq|r| \leq N-q+\nu \\ & 0 \leq|r| \leq N+q \\ 0 & \text { otherwise. }\end{cases}
$$

Using (12), we find after tedious manipulations of (7)-(9), the following simple expressions:

$$
\begin{gathered}
P_{U}=|\alpha|^{2}|\Phi(0)|^{2}+\frac{1}{N} \sum_{k=-\infty}^{+\infty} \sum_{\ell=-\infty}^{+\infty} w(k ; \ell) R_{M P}(k ; \ell) \\
P_{I C I}=|\alpha|^{2}+\sum_{k=-\infty}^{+\infty} w(k ; 0) R_{M P}(k ; 0)-P_{U} \\
P_{I S I}=\sum_{k=-\infty}^{+\infty}(1-w(k ; 0)) R_{M P}(k ; 0)
\end{gathered}
$$

where $\Phi(0)$ is the discrete Fourier transform of length $N$ of the LOS phase $\phi(\ell)$ at frequency 0 . For example, assuming that the phase rotation of the LOS component is caused by a Doppler shift $f_{D}$, i.e. $\phi(\ell)=\phi(0)-2 \pi f_{D} \ell T$, with $1 /(N T)$ the carrier spacing, $|\Phi(0)|^{2}$ reduces to

$$
|\Phi(0)|^{2}=\left|\frac{\sin \left(\pi N f_{D} T\right)}{N \sin \left(\pi f_{D} T\right)}\right|^{2} .
$$

Note that, because of the assumption that $P_{L O S}+P_{M P}=1$, the sum of the powers of the useful component, the ICI and ISI equals one: $P_{U}+P_{I C I}+P_{I S I}=1$. In the absence of a LOS component (i.e. $\alpha=0$ ), the equations (13)-(15) reduce to the expressions in [6]. 
As can be observed in (13)-(15), the useful power and the ICI power depend on both the LOS component and the multipath component, whereas the ISI power only depends on the multipath component. This can easily be explained, as the channel $h_{L O S}(k ; \ell)$ consists of a single tap and hence will not cause interference between successive multicarrier blocks. Let us consider quantitatively the effect of the system parameters $N$ and $\nu$ on the performance of the system. We separately consider the effect of the system parameters on the powers originating from the LOS component and the multipath component.

- LOS contribution: The useful power originating from the LOS component, i.e. $P_{U, L O S}=|\alpha|^{2}|\Phi(0)|^{2}$ will reduce for increasing $N$, as $|\Phi(0)|^{2}$ is in general a decreasing function of $N$. This can be seen in (16) for the case of a Doppler shift. As $h_{L O S}(k ; \ell)$ consists of a single tap, there is no ISI, i.e. $P_{I S I, L O S}=0$, such that the interference consists only of ICI. The ICI power corresponding to the LOS component, i.e. $P_{I C I, L O S}=$ $|\alpha|^{2}\left(1-|\Phi(0)|^{2}\right)$ will increase for increasing $N$. On the other hand, the powers of the useful component and the ICI corresponding to the LOS component do not depend on the cyclic prefix length $\nu$. However, increasing the cyclic prefix length will reduce the power efficiency through the factor $\frac{N}{N+\nu}$, as only $N$ of the $N+\nu$ samples are used for further processing. Hence, from the LOS component point of view, the cyclic prefix must be as small as possible, and because of the time-selectivity of the LOS component, the length $N$ of the multicarrier symbol must be limited, as increasing $N$ will reduce the performance.

- Multipath contribution: For given $N$, increasing the length of the cyclic prefix will reduce the interference power $P_{I C I}+P_{I S I}$, as the distortion of the $N$ samples that are processed by the receiver reduces. At the same time, the useful power moves closer to 1 . However, because of the factor $\frac{N}{N+\nu}$ the power efficiency will reduce. For given $\nu$, increasing $N$ will reduce the relative importance of the fixed amount of distorted samples (caused by the frequency selectivity of the channel) at the edges of the block of $N$ samples that is further processed. Further, increasing $N$ will increase the power efficiency as the factor $\frac{N}{N+\nu}$ moves closer to one. However, increasing $N$ will result in longer multicarrier symbols, such that the system will suffer from interference caused by the time selectivity of the channel.

Taking into account these considerations, it is clear that an optimum set of system parameters $\left(N_{o p t}, \nu_{o p t}\right)$ can be found Further, for a given shape of the LOS and multipath channel impulse responses, the ratio $\kappa$ of the powers of the LOS contribution to the multipath contribution will have an effect on the optimal parameters. For increasing $\kappa$, i.e. for channels with stronger LOS component, the optimum parameters will move to $N_{o p t}=1$ and $\nu_{o p t}=0$, which are the optimum system parameters when there is no multipath component.

\section{LiMiting CASES}

As observed in (13), the useful power (and thus also the ICI power in (14)) corresponding to the multipath component consists of a double sum, which requires a high computational complexity, especially when $N$ is large. To reduce the computational complexity, we have shown in [6] for multipath Rayleigh fading channels that the useful power and interference powers can be well approximated by considering two limiting cases, i.e. the time-flat and frequency-flat fading Rayleigh fading channels derived from the considered Rayleigh fading channel, and take the sum of the powers of the limiting cases to obtain the total useful power and interference powers.

The same reasoning can be followed for the channel considered in this paper. As we observe in (13)-(15), the powers of the useful component, the ICI and ISI consist of the sum of the powers originating from the LOS component and the multipath component. Further, taking into account the results from [6], the powers corresponding to the multipath component can be further split into the sum of the powers corresponding to the limiting cases of a time-flat channel with autocorrelation function $R_{M P, T F}(k ; \ell)=R_{M P}(k ; 0)$ and a frequencyflat channel with autocorrelation function $R_{M P, F F}(k ; \ell)=$ $\tilde{R}_{M P, F F}(\ell) \delta(k)$, where $\tilde{R}_{M P, F F}(\ell)=\sum_{k=-\infty}^{+\infty} R_{M P}(k ; \ell)$. Hence, we approximate the powers in (13)-(15) using the following decomposition: $P_{X}=P_{X, L O S}+P_{X, M P, T F}+$ $P_{X, M P, F F}$, with $X=U, I C I$ and $I S I$; the definition of $P_{X, L O S}$ can be found in the previous section. The useful powers corresponding to the two limiting cases of the multipath component are given by [6]:

$$
\begin{array}{r}
P_{U, M P, T F}=\sum_{k=-\infty}^{+\infty} w^{2}(k ; 0) R_{M P}(k ; 0) \\
P_{U, M P, F F}=\frac{1}{N} \sum_{\ell=-N}^{N}\left(1-\frac{|\ell|}{N}\right) \tilde{R}_{M P, F F}(\ell)
\end{array}
$$

and the derivation of the corresponding interference powers follow straightforward from (13)-(15) and the definition of $R_{M P, T F}(k ; \ell)$ and $R_{M P, F F}(k ; \ell)$. In the next section, we will show the validity of the used approximations by means of numerical evaluation.

\section{NuMERICAL RESUltS}

For the numerical evaluation of the obtained analytical expressions, we consider a channel bandwidth $B=1 \mathrm{MHz}$ and a center frequency of $f_{c}=1 \mathrm{GHz}$. Hence, the duration of a sample equals $T=1 \mu \mathrm{s}$. For the LOS component of the channel, we assume that the channel has a fixed amplitude $\alpha$ and a phase rotation $\phi(\ell)$ caused by a Doppler shift $f_{D}$, i.e. $\phi(\ell)=\phi(0)-2 \pi f_{D} \ell T$. Assuming that the receiver is moving at a speed of $v=135 \mathrm{~km} / \mathrm{hr}$, the Doppler shift is given by $f_{D}=(v / c) f_{c}=125 \mathrm{~Hz}$, where $c$ is the velocity of light. For the multipath component of the channel, we use the typical urban (TU) [7]-[8] impulse response with a delay spread of $5 \mu \mathrm{s}$. The coherence time of the multipath component is obtained using the rule of thumb $T_{0}=0.5 / f_{D}$ [9], where $f_{D}$ is the Doppler frequency of the LOS component, resulting in the coherence time $T_{0}=4 \mathrm{~ms}$. We assume that the autocorrelation function for the multipath component has an exponentially decaying multipath intensity profile and a Gaussian time correlation profile

$$
\begin{array}{r}
R_{M P}(k ; \ell)=C \exp \left(-\frac{k}{\tau_{0}}\right) \exp \left(-\frac{\ell^{2}}{2 \sigma_{0}^{2}}\right) \\
k \geq 0,-\infty \leq \ell \leq+\infty
\end{array}
$$

Defining the delay spread as the time at which the multipath intensity profile falls $10 \mathrm{~dB}$ below the level of the strongest component, the parameter $\tau_{0}$ is found to be about five samples. Further, we fix the coherence time $T_{0}$ to twice the duration of the spreading of the Gaussian time correlation profile, i.e. 


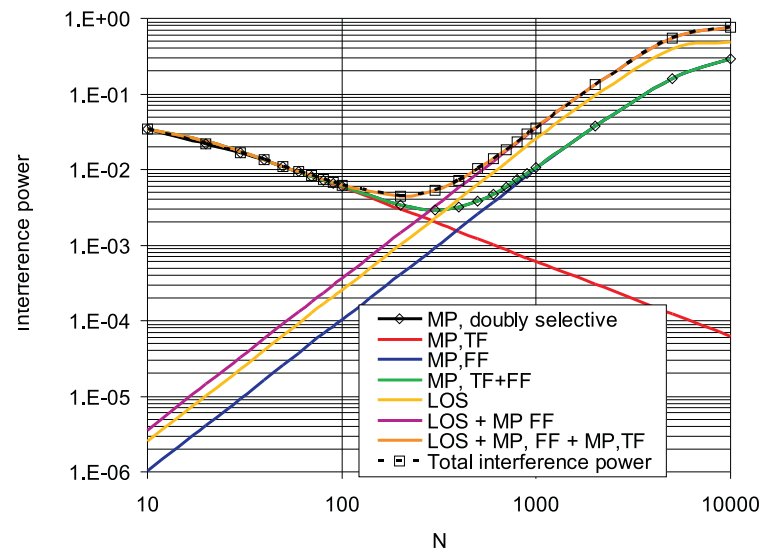

Fig. 2. Interference power as function of FFT length, $\kappa=0 \mathrm{~dB}, \nu=10$.

$\sigma_{0}=2000$ samples. The constants $\alpha$ and $C$ are normalization constants.

In figure 2, we show the total interference power $P_{I C I}+$ $P_{I S I}$, obtained with (14) and (15), together with the interference power of the limiting cases and the sum of the interference powers of the limiting cases as function of $N$, for $\kappa=0 \mathrm{~dB}$ and $\nu=10$. As expected, the interference power corresponding to the LOS component increases with increasing $N$. For $N f_{D} T \ll 1$, i.e. when the Doppler shift is sufficiently smaller than the carrier spacing, the approximation $\left|\frac{\sin \left(\pi N f_{D} T\right)}{N \sin \left(\pi f_{D} T\right)}\right|^{2} \approx 1-\frac{1}{3}\left(\pi N f_{D} T\right)^{2}$ can be used, such that the interference power for the LOS component, given by $|\alpha|^{2}\left(1-|\Phi(0)|^{2}\right)$, increases quadratically with $N$. In [6], it was shown that for a multipath fading channel the total interference power for the time-flat channel with autocorrelation function $R_{M P, T F}(k ; \ell)=R_{M P}(k ; 0)$ is proportional to $1 / N$. Further, in the case of the frequency-flat limiting case of the multipath channel, when the autocorrelation function $R_{M P, F F}(k ; \ell)=\tilde{R}_{M P, F F}(\ell) \delta(k)$ can be approximated ${ }^{2}$ by $\tilde{R}_{M P, F F}(\ell) \approx \tilde{R}_{M P, F F}(0)\left(1-\beta \ell^{2}\right)$ for $\ell \ll T_{0}$, it can easily be shown that the total interference power increases with $N^{2}$. This can be observed in figure 2 . Figure 2 also shows the total interference power of the multipath component (indicated in the figure as MP, doubly selective), obtained with the contributions of $R_{M P}(k ; \ell)$ in (14) and (15). This interference power is well approximated by the sum of the interference powers (indicated in the figure as $\mathrm{MP}, \mathrm{TF}+\mathrm{FF}$ ) corresponding to the time-flat and frequency-flat limiting cases [6]. Further, we show in figure 2 the sum of the interference powers of the LOS component and the limiting cases of the multipath component (indicated as LOS + MP,FF + MP,TF), and the total interference power obtained with (14) and (15). As we observe, the sum of the interference powers of the limiting cases well approximate the total interference power of the actual doubly selective channel with LOS component. For small values of $N$, the interference power of the actual channel converges to the interference power of the time-flat limit of the multipath component, whereas for large $N$, it converges to the sum of the interference powers of the LOS component and the frequency-flat limit of the multipath component (indicated as $\mathrm{LOS}+\mathrm{MP}, \mathrm{FF})$.

In figure 3, the interference power of the actual channel, the limiting cases and the sum of the interference powers of

${ }^{2}$ In most practical channels, this approximation is valid.

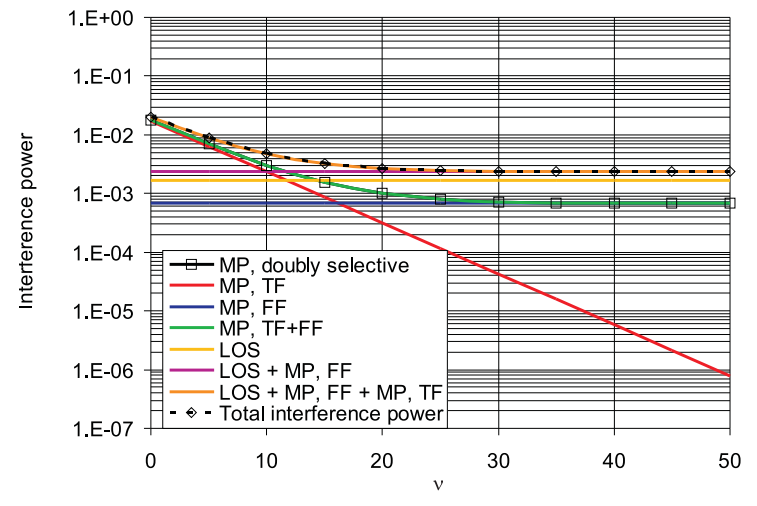

Fig. 3. Interference power as function of cyclic prefix length, $\kappa=0 \mathrm{~dB}$, $N=256$

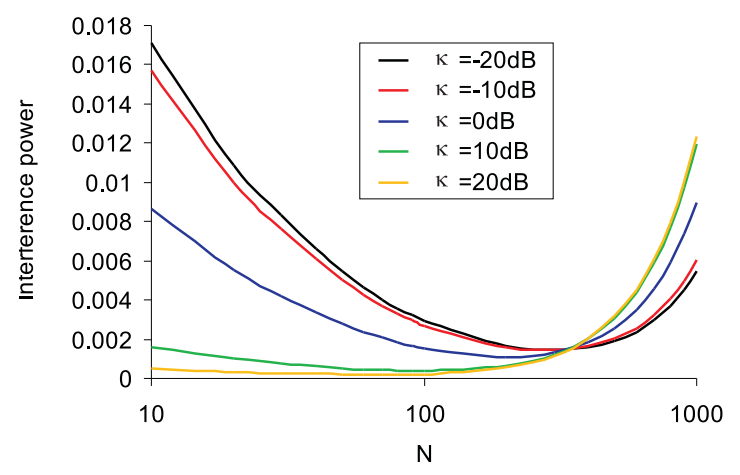

Fig. 4. Influence of $N$ and $\kappa$ on interference power, $\nu=10$.

the limiting cases are shown as function of $\nu$, for $\kappa=0$ $\mathrm{dB}$ and $N=256$. Similarly as in figure 2 , the sum of the interference powers of the limiting cases well approximate the interference power of the actual channel. As expected, the interference powers of the frequency-flat limit of the multipath component and the LOS component are independent of the length of the cyclic prefix. The interference power of the time-flat limit of the multipath component is a decreasing function of $\nu$ as the effect of the channel dispersion reduces for increasing $\nu$; the slope of the decreasing function will depend on the shape of the multipath intensity profile. For small $\nu$, the interference power of the actual channel converges to the interference power of the time-flat limit of the multipath component, whereas for large $\nu$, the interference power of the actual channel no longer decreases but converges to the sum of the interference powers of the LOS component and the frequency-flat limit of the multipath component; the resulting interference is caused by the time-selectivity of the channel and cannot be combatted by a further increase of the cyclic prefix length.

Figures 4 and 5 show the effect of $\kappa$, indicating the relative amount of power contained in the LOS component and the multipath component, on the interference power. As can be observed in figure 4 , the minimum of the interference power moves to lower values of $N$ when $\kappa$ increases. This can be explained as follows. When $\kappa$ increases, the LOS component becomes the dominating component. As in this case the interference is caused by the time-selectivity of the LOS 


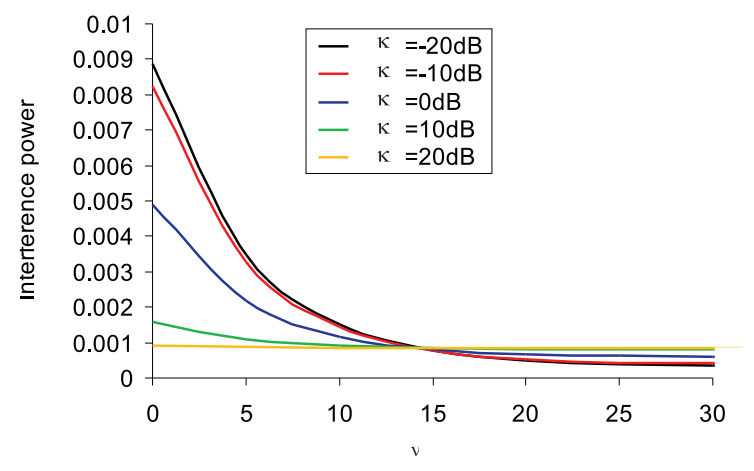

Fig. 5. Influence of $\nu$ and $\kappa$ on interference power, $N=256$.

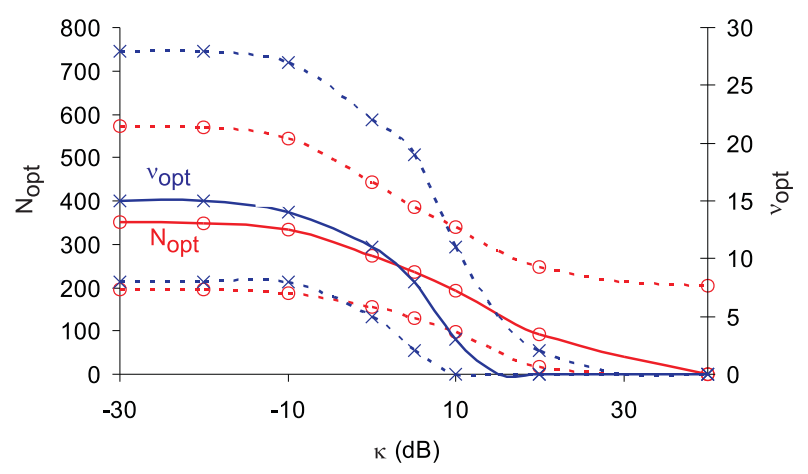

Fig. 6. Optimal $N$ and $\nu$ and range for $N$ and $\nu$ for which degradation $\leq 0.1 \mathrm{~dB}$ larger than minimum degradation, $E_{s} / N_{0}=10 \mathrm{~dB}$.

component, the interference will increase when $N$ increases, resulting in a reduction of the optimum value of $N$. The effect of $\kappa$ on the dependency of the interference power on $\nu$ is shown in figure 5. As for large $\kappa$, the channel consists of mainly LOS, and the relative importance of the frequency selectivity decreases, the interference power becomes essentially independent of $\nu$, whereas for small values of $\kappa$, when the multipath component is dominating, the interference power strongly depends on the value of $\nu$.

These effects can also be observed in figure 6 , where the optimum value of $N$ and $\nu$ that minimize the degradation (11) are shown as function of $\kappa$. Similarly as in figure 4 we observe that $N_{\text {opt }}$ decreases for increasing $\kappa$. At the same time, $\nu_{\text {opt }}$ decreases for increasing $\kappa$; as the interference power for large $\kappa$ becomes essentially independent of $\nu$, this reduction of $\nu_{o p t}$ will be caused by the power efficiency factor $\frac{N}{N+\nu}$. Further, in figure 6, the range over which $N(\nu)$ given $\nu_{\text {opt }}$ $\left(N_{o p t}\right)$ may vary such that the degradation is smaller than the minimum degradation plus $0.1 \mathrm{~dB}$, is shown (dashed curves). The range over which $N$ or $\nu$ may vary is large for small $\kappa$ and reduces when $\kappa$ increases. This is explained as follows. It is obvious that, as $\nu_{o p t} \rightarrow 0$ when $\kappa$ increases and as $\nu$ cannot be negative, the lower range for $\nu$ decreases when $\kappa$ increases. Further for large $\kappa$, the interference power is small (see figures 4 and 5) when $N \leq N_{\text {opt }}$. In this case, the degradation is mainly caused by the power efficiency factor $\frac{N}{N+\nu}$ in the first term of (11). As $N_{o p t}$ and $\nu_{o p t}$ are rather small, the factor $\frac{N}{N+\nu}$ strongly varies as function of $N$ and $\nu$, such that the degradation will strongly increase when $N$ decreases or $\nu$ increases, hence the lower range for $N$ and the upper range for $\nu$ become smaller when $\kappa$ increases. On the other hand, when $N>N_{\text {opt }}$, the interference power increases significantly (see figure 4). Hence, the beneficial effect of the increase of $N$ in the factor $\frac{N}{N+\nu}$ will be counteracted by the increase of the interference power, such that the upper range for $N$ only changes minimally. As a result of this, the selection of the parameters $N$ and $\nu$ is less critical when there is a large multipath component as compared to the case of a large LOS component.

\section{CONCLUSIONS}

In this paper, we have investigated the effect of the system parameters, i.e. the cyclic prefix length and the number of carriers, on the performance of an multicarrier system in a doubly selective fading channel with LOS component. We have derived analytical expressions for the degradation. Further, we have found simple but accurate approximations for the power of the useful component and the interference, by decomposing the doubly selective fading channel with LOS component into three limiting cases, i.e. a time-flat and a frequency-flat Rayleigh fading channel and a channel with only LOS, and taking the sum of the powers of the three limiting cases as approximation for the powers of the actual channel. Using these analytical expressions, the optimal system parameters $\left(N_{o p t}, \nu_{o p t}\right)$ that minimize the degradation, are obtained. Further, the range over which the system parameters $N$ and $\nu$ may vary when a small degradation $(0.1 \mathrm{~dB})$ as compared to the minimum degradation is allowed has been determined. Moreover, the influence of the channel parameter $\kappa$, i.e. the ratio of the powers contained in the LOS component and the multipath component of the channel on the optimum parameters and deviations from the optimum parameters has been studied. The results can be summarized as follows. When $\kappa$ increases, i.e. when the LOS component is the dominating component, the values of $N_{o p t}$ and $\nu_{o p t}$ decrease. Further, as the range over which the system parameters may vary when a small degradation is allowed as compared to the minimum degradation decreases, the selection of the system parameters $N$ and $\nu$ becomes more critical when $\kappa$ increases.

\section{ACKNOWLEDGMENT}

This work has been supported by the Interuniversity Attraction Poles Program - Belgian State - Federal Office for Scientific, Technical and Cultural Affairs.

\section{REFERENCES}

[1] J. A. C. Bingham, "Multicarrier modulation for data transmission, an idea whose time has come," IEEE Commun. Mag., vol. 31, pp. 514, May 1990.

[2] Z. Wang, G.B. Giannakis, Wireless Multicarrier Communications, IEEE Signal Processing Magazine, Vol. 17, No. 3, pp. 29-48, May 2000.

[3] CJ Park, GH Im, "Efficient DMT/OFDM transmission with insufficient cyclic prefix", IEEE Communications Letters, Vol. 8, No. 9, pp. 576578, Sep 04

[4] S. Chen, C. Zhu,'ICI and ISI analysis and mitigation for OFDM systems with insufficient cyclic prefix in time-varying channels", IEEE Transactions on Consumer Electronics, Vol. 50, No.1, pp 78-83, Feb 2004.

[5] F. Tufvesson and T. Maseng, "Optimization of sub-channel bandwidth for mobile OFDM systems," in Proc. Multiaccess, Mobility and TeletrafficAdvances in Wireless Networks (MMT), 1998, pp. 103114.

[6] H. Steendam, M. Moeneclaey, "Analysis and Optimization of the Performance of OFDM on Frequency-Selective Time-Selective Fading Channels", IEEE Trans. on Comm., vol. 47, no. 12, pp. 1811-1819, Dec 1999.

[7] R. Steele, Mobile Radio Communications. London, U.K.: Pentech, 1992.

[8] K. Pahlavan and A. H. Levesque, Wireless Information Networks, New York: Wiley, 1995, ch. 6.

[9] B. Sklar, "Rayleigh fading channels in mobile digital communication systems, part I: Characterization," IEEE Commun. Mag., vol. 38, pp. 90100, July 1997. 